\title{
Interhemispheric Relationship of Genetic Influence on Human Brain Connectivity
}

\author{
Suyu Zhong ${ }^{1}$, Long $\mathrm{Wei}^{2}$, Chenxi Zhao ${ }^{1}$, Liyuan Yang ${ }^{1}$, Zengru $\mathrm{Di}^{3}$, \\ Clyde Francks ${ }^{4,5}$ and Gaolang Gong ${ }^{1,6}$
}

\begin{abstract}
${ }^{1}$ State Key Laboratory of Cognitive Neuroscience and Learning \& IDG/McGovern Institute for Brain Research, Beijing Normal University, Beijing 100875, China, ${ }^{2}$ School of Computer Science and Technology, Shandong Jianzhu University, Jinan, Shandong 250101, China, ${ }^{3}$ School of Systems Science, Beijing Normal University, Beijing 100875, China, ${ }^{4}$ Language and Genetics Department, Max Planck Institute for Psycholinguistics, 6525 XD Nijmegen, The Netherlands, ${ }^{5}$ Donders Institute for Brain, Cognition and Behavior, Radboud University, 6525 EN Nijmegen, The Netherlands and ${ }^{6}$ Beijing Key Laboratory of Brain Imaging and Connectomics, Beijing Normal University, Beijing 100875, China
\end{abstract}

Address correspondence to Dr Gaolang Gong, State Key Laboratory of Cognitive Neuroscience and Learning, Beijing Normal University, \#19 Xinjiekouwai Street, Beijing 100875, China. Email: gaolang.gong@bnu.edu.cn.

S.Z. and L.W. contributed equally to this work.

\begin{abstract}
To understand the origins of interhemispheric differences and commonalities/coupling in human brain wiring, it is crucial to determine how homologous interregional connectivities of the left and right hemispheres are genetically determined and related. To address this, in the present study, we analyzed human twin and pedigree samples with high-quality diffusion magnetic resonance imaging tractography and estimated the heritability and genetic correlation of homologous left and right white matter (WM) connections. The results showed that the heritability of WM connectivity was similar and coupled between the 2 hemispheres and that the degree of overlap in genetic factors underlying homologous WM connectivity (i.e., interhemispheric genetic correlation) varied substantially across the human brain: from complete overlap to complete nonoverlap. Particularly, the heritability was significantly stronger and the chance of interhemispheric complete overlap in genetic factors was higher in subcortical WM connections than in cortical WM connections. In addition, the heritability and interhemispheric genetic correlations were stronger for long-range connections than for short-range connections. These findings highlight the determinants of the genetics underlying WM connectivity and its interhemispheric relationships, and provide insight into genetic basis of WM connectivity asymmetries in both healthy and disease states.
\end{abstract}

Key words: brain asymmetry, genetic correlation, heritability, homology, WM connectivity

\section{Introduction}

The differences and commonalities between the 2 brain hemispheres are of general interest in neuroscience. By focusing on interhemispheric differences, numerous studies have demonstrated the existence of structural asymmetries and functional lateralization in various brain phenotypes (Toga and Thompson 2003; Bishop 2013; Herve et al. 2013; Kong et al. 2018). On the other hand, the commonalities and coupling between the 2 hemispheres are obvious: the left and right hemispheres largely mirror each other; the developmental and aging-related changes that occur in homologous left and right brain structures are highly synchronized (Scott et al. 2012; Andescavage et al. 2016).

To understand the origin of interhemispheric differences and commonalities/couplings, it is crucial to determine how 
homologous left and right brain structures are genetically related. This involves 2 related questions: 1) Are homologous left and right brain structures affected to similar degrees by genetic influences? 2) To what degree do overlapping genetic factors affect homologous left and right brain structures? Neuroimaging findings have provided important clues to both questions. Relevant to the first question, while a few studies have reported that the degree of heritability of gray matter (GM) morphometric phenotypes is stronger in the regions/lobes of one hemisphere than in the other hemisphere (Geschwind et al. 2002; Yoon et al. 2010), many other studies have concluded no interhemispheric differences in heritability (Wright et al. 2002; Kremen et al. 2010; Eyler et al. 2014; Wen et al. 2016; Liu et al. 2019). Regarding the second question, a number of studies have applied analyses of genetic correlations to measure degrees of overlap in genetic factors between 2 phenotypes, and the majority of them have consistently shown that there is great overlap in the genetic factors that underlie the various GM phenotypes of homologous left and right regions/lobes (Eyler et al. 2014; Guadalupe et al. 2016; Kong et al. 2018; Liu et al. 2019).

However, the relevant findings in previous studies are limited to GM phenotypes. Very few studies have focused on the genetic relationships between homologous left and right white matter (WM) phenotypes, although the heritability of voxelwise or tractwise WM imaging parameters, such as volume and fractional anisotropy, has been explored (Chiang et al. 2011; Kochunov et al. 2014; Kochunov et al. 2015; Lee et al. 2015). Interregional WM connectivity represents an intuitive measurement of the strength of anatomical connections between $2 \mathrm{GM}$ regions of interest and has been widely applied in the context of brain connectome research (Gong et al. 2009; Sporns 2011; Sotiropoulos and Zalesky 2019). Surprisingly, the genetics of this important WM phenotype remain very poorly explored. As a consequence, the mechanisms by which the homologous left and right WM connectivities of the 2 hemispheres are genetically determined and related remain largely unknown. Understanding these particular genetics is a prerequisite for detangling the genetic basis of observed interhemispheric similarities and asymmetries in human brain connectivity and the human brain connectome (Thompson et al. 2013; Zhong et al. 2017; Zhao et al. 2019b).

The present study sought to exclusively assess the genetics of interregional WM connectivity with a focus on genetic relationships between the 2 hemispheres. Given previous findings related to GM phenotypes, we first hypothesized that the heritability of homologous WM connectivity would be similar between the 2 hemispheres and that the degree of overlap in genetic factors would be high between homologous left and right WM connections. Given that the functions of short- and long-range WM connections are putatively distinct at the system level (Kaiser and Hilgetag 2006; Betzel and Bassett 2018), we further hypothesized that short- and longrange WM connections differ in the heritability of phenotypes and interhemispheric genetic correlations. To test these hypotheses, we used diffusion magnetic resonance imaging (MRI) acquisition of the Human Connectome Project (HCP) dataset to estimate the interregional WM connectivity of $\sim 1000$ subjects and then applied twin and pedigree information to estimate the heritability of and genetic correlations between the homologous WM connections in both hemispheres.

\section{Materials and Methods}

\section{Subjects}

The HCP dataset was used in this study (Van Essen et al. 2012). In total, 930 healthy adults from the HCP S1200 release for whom both diffusion and T1 images were available were included. The included subjects aged 22-37 years old (28.7 \pm 3.7 years; 426 males and 504 females). The zygosity and parental identities of the included individuals were confirmed by genomic data obtained using blood or saliva samples. The 930 HCP subjects included 107 monozygotic twin pairs with their 77 siblings and 3 half-siblings, 54 dizygotic twin pairs with their 47 siblings and 5 half-siblings, 356 siblings, 10 half-siblings, and 110 unrelated individuals. All subjects provided written informed consent, and the research protocol was approved by the Institutional Review Board of Washington University.

\section{MRI Acquisition and Preprocessing}

MRIs were acquired using a Siemens Connectome Skyra $3 \mathrm{~T}$ scanner housed at Washington University in St. Louis. The diffusion-weighted images (DWI) were collected using an HCPspecific variant of the multiband diffusion sequence. For the diffusion images, 3 different gradient tables were used. Each table was acquired once with right-to-left and left-to-right phase-encoding polarities and included 90 diffusion-weighting directions plus $6 b=0$ images interspersed throughout each run. The DWI consisted of 3 shells of $b=1000,2000$ and $3000 \mathrm{~s} / \mathrm{mm}^{2}$ interspersed with an equal number of acquisitions on each shell within each run. The detailed parameters were as follows: time repetition $(\mathrm{TR})=5520 \mathrm{~ms}$, time echo $(\mathrm{TE})=89.5 \mathrm{~ms}$, flip angle $=78^{\circ}$, refocusing flip angle $=160^{\circ}$, field of view $(F O V)=210 \times 180 \mathrm{~mm}^{2}$, matrix $=168 \times 144$, slice thickness $=1.25$ $\mathrm{mm}$ (111 slices), and multiband factor $=3$. For $\mathrm{T}_{1}$-weighted images, 256 slices per slab were acquired with a 3D magnetizationprepared rapid gradient echo sequence as follows: $T R=2400 \mathrm{~ms}$, $\mathrm{TE}=2.14 \mathrm{~ms}$, time to inversion $=1000 \mathrm{~ms}$, flip angle $=8^{\circ}$, FOV $=224 \times 224 \mathrm{~mm}^{2}$, and resolution $=0.7 \times 0.7 \times 0.7 \mathrm{~mm}^{3}$. DWI and $\mathrm{T}_{1}$-weighted images were preprocessed using the HCP minimal-preprocessing pipelines (Glasser et al. 2013).

\section{Diffusion MRI-Based Tractography}

For each voxel, fiber orientation distributions (FODs) were estimated using multishell multitissue constrained spherical deconvolution (Tournier et al. 2007; Jeurissen et al. 2014). Here, the single-fiber WM response function as well as the GM and cerebrospinal fluid (CSF) response functions were estimated using the dhollander algorithm with a harmonic order of 8 (Dhollander et al., 2016). Whole-brain tractography was then carried out in the individual-specific diffusion MRI space by using the second-order integration over fiber orientation distributions (iFOD2) algorithm in the MRtrix3 package (Tournier et al. 2012; Tournier et al. 2019). To improve the biological accuracy of fiber reconstruction during tracking, we used the anatomically constrained tractography (ACT) framework, in which anatomical prior information can be effectively considered (Smith et al. 2012). Here, the anatomical prior information was obtained by segmenting the T1 images. Given that the HCP preprocessed T1 images were well aligned with the DWI, we did not need to coregister the 2 images for each subject. Specifically, the T1 image was segmented into tissue 
partial volume maps (PVMs) of the brain WM, GM and CSF using FMRIB Software Library tools (Smith et al. 2004). The resultant PVM images were then taken as anatomical priors into the ACT framework. The detailed fiber-tracking parameters were as follows: step size $=0.625 \mathrm{~mm}$, maximum curvature per step $=45^{\circ}$, FOD amplitude threshold $=0.06$, minimum length $=2.5 \mathrm{~mm}$, and maximum length $=250 \mathrm{~mm}$. For each individual, 100 million streamlines were initially generated by seeding randomly from the ACT-generated GM/WM interface, and these streamlines were subsequently filtered to obtain 10 million streamlines through the spherical-deconvolution informed filtering of tractograms method. This postfiltering process can effectively improve the quantitative nature of all reconstructed streamlines (Smith et al. 2013).

\section{Interregional WM Connectivity}

Two atlases in the Montreal Neurological Institute (MNI) space were applied to parcellate the entire GM into a set of regions. The first was the Brainnetome atlas (BNA), which was derived from WM connectional information (Fan et al. 2016). Specifically, this atlas includes 246 cerebral regions (210 cortical and 36 subcortical GM regions, with half of each from each hemisphere), and the voxels within each parcellated region were considered putatively homogenous in terms of the whole-brain WM connectional profile. The second was the atlas of intrinsic connectivity of homotopic areas (AICHA) (Joliot et al. 2015). This particular functional atlas takes the regional homotopy between the 2 hemispheres into account when parcellating GM regions with resting-state functional connectivity. It includes 384 cerebral regions (340 cortical and 44 subcortical GM regions, with half of each from each hemisphere). To obtain the BNA/AICHA parcellation for each individual-specific diffusion MRI space, the MNI-to-T1 nonlinear transformations derived from the HCP minimal-preprocessing pipelines were applied to the original BNA/AICHA of the MNI space.

For each individual, the interregional WM streamlines connecting each pair of BNA or AICHA regions within each hemisphere were determined using the whole-brain tractogram described above (Fig. 1). Each streamline was assigned to the closest region within a sphere with a $2-\mathrm{mm}$ radius centered at its endpoint, and the set of streamlines linking each BNA or AICHA regional pair was then derived from the tractogram (Tournier et al. 2012, 2019). Given the large-scale nature of BNA/AICHA regions, it is plausible to assume a consistent yes-no pattern for the existence of fibers between each pair of regions across all subjects. The observation of streamlines linking a particular pair of regions in only a portion of subjects was likely to be spurious (e.g., due to noisy data and algorithm errors), and these streamlines were therefore discarded in those subjects (Zhao et al. 2019b). This is equivalent to applying a threshold of $100 \%$ to the subject proportion of nonzero connections for every 2 regions. A set of WM connections was then obtained for each atlas (BNA connections: 2467 for the left and 2313 for the right; AICHA connections: 3351 for the left and 3457 for the right). Furthermore, to ensure comparability between the 2 hemispheres, we confined our analysis to interregional WM connections found in both hemispheres (BNA: 2035 withinhemispheric connections; AICHA: 2840 within-hemispheric connections). For each of the interregional WM connection, the strength/weight (i.e., WM connectivity) was defined as the fraction of streamlines (FSe), which is analogous to the definition of connection strength used in retrograde-tracerbased studies. Strong correlations between this diffusion MRI-derived measure and tracer-based connection strength have been verified in monkey brains (Donahue et al. 2016). Specifically, the FSe was calculated as the fraction of streamlines that linked 2 regions relative to the total number of streamlines extrinsic to those regions (Donahue et al. 2016; Zhao et al. 2019b).

\section{Short- and Long-Range Definitions of Interregional WM Connections}

For each interregional WM connection within a hemisphere, the length was estimated in 2 ways: 1) the mean length of the tractogram-derived streamlines between the 2 linked BNA/AICHA regions across all subjects and 2) the Euclidean distance between the 2 linked BNA/AICHA regions on the atlas of the MNI space. For both methods, the length of the homologous left and right WM connection was highly correlated (streamline-based, BNA: $R=0.93, P<10^{-15}$; AICHA: $R=0.94, P<10^{-15}$; Euclidean distance-based, BNA: $R=0.98$, $P<10^{-15}$; AICHA: $\left.R=0.99, P<10^{-15}\right)$, and we therefore averaged the left and right measurements to obtain a single length for each pair of homologous WM connections. To provide a straightforward illustration of the effect of length on the genetics of interregional WM connectivity, we used this single length value to categorize all interregional WM connections into 1) short-range connections (length $<$ mean of all connections) or 2) long-range connections (length $>$ mean of all connections).

Based on its linked BNA/AICHA regions, each WM connection was also categorized as anatomically within- or between-system (7 classical anatomical systems were defined as follows: the frontal lobe, temporal lobe, partial lobe, occipital lobe, insular lobe, limbic system, and subcortical nuclei). This categorization was taken as another indirect approach to define the length of WM connections, with within- and between-system connections corresponding to short- and long-range connections, respectively.

\section{Quantitative Genetic Estimation and Statistical Analysis}

In the present study, we mainly focused on 2 types of genetic estimation: 1) the heritability of WM connectivities and 2) the genetic correlations between the homologous left and right WM connectivities of the 2 hemispheres.

\section{Heritability}

For any phenotype, heritability represents the proportion of phenotypic variance that can be explained by genetic variance among individuals within a population and is typically referred to as $h^{2}$. For each WM connection (BNA: 2035 connections in total for each hemisphere; AICHA: 2840 connections in total for each hemisphere), $h^{2}$ was estimated after controlling for age, sex, and hemispheric intracranial volume (ICV). Here, we controlled for the hemispheric ICV because this highly heritable trait showed a strong association with WM connectivity and therefore may confound our genetic estimation for WM connectivity (Hänggi et al. 2014; Jansen et al. 2015). To determine whether the heritability of the left WM connectivity is significantly different from that of its homologous right WM connectivity, we used a confidence interval (CI)-based approach. Specifically, under the asymptotic normality assumption of the maximum likelihood estimator, the CI at $100(1-\alpha) \%$ for the estimated heritability is $\left[h^{2}-Z(\alpha / 2) \times \sigma, h^{2}+Z(\alpha / 2) \times \sigma\right]$, where $\sigma$ is the standard 

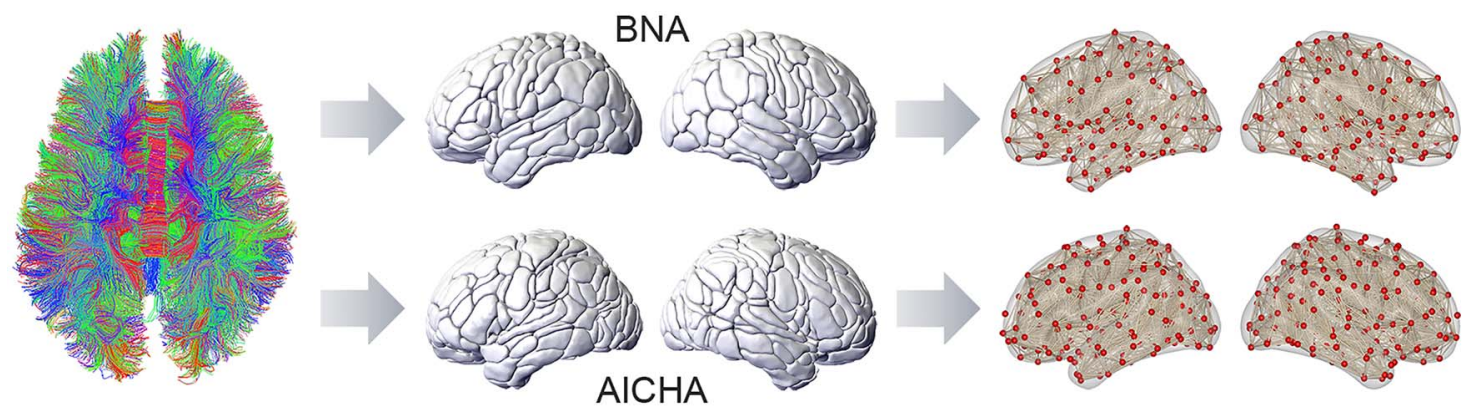

Figure 1. Schematic overview of the extraction of interregional WM connections. Whole-brain tracts were first estimated using diffusion MRI-based tractography. Two atlases were applied to parcellate the entire cerebral GM into a set of regions: BNA, the Brainnetome atlas (Fan et al. 2016), and AICHA (Joliot et al. 2015).

error of the estimated heritability (Neale and Miller 1997). For 2 homologous WM connections, the two $h^{2}$ values were therefore considered different at the significance level of $100(1-\alpha) \%$, if $\left|h^{2}{ }_{\text {right }}-h^{2}{ }_{\text {left }}\right|>Z(\alpha / 2) \times\left(\sigma_{\text {right }}+\sigma_{\text {left }}\right)$ (Eyler et al. 2014; Le Guen et al. 2018).

\section{Genetic Correlation}

To estimate the degree of overlap in genetic factors underlie homologous WM connectivity in the 2 hemispheres, we calculated the genetic correlation coefficient $\left(r_{\mathrm{g}}\right)$ for each pair of homologous WM connections (BNA: 2035 connection pairs in total; AICHA: 2840 connection pairs in total). Specifically, the $r_{\mathrm{g}}$ between 2 phenotypes is defined as the genetic covariance divided by the square root of the product of their separate genetic variances (Neale and Cardon 1992). The $r_{\mathrm{g}}$ value ranges from -1 to 1 , and positive and negative values indicate the same or opposite functioning direction, respectively, of overlapped genetic factors to the 2 phenotypes. In this equation, $r_{\mathrm{g}}=1$ or -1 indicates that genetic factors show complete overlap between the 2 phenotypes, whereas $r_{\mathrm{g}}=0$ indicates a complete lack of overlap in genetic factors between the 2 phenotypes (Almasy et al. 1997). In the present study, the $r_{g}$ for each pair of homologous WM connections was calculated after controlling for age and sex.

All these genetic estimations were carried out using the Sequential Oligogenic Linkage Analysis Routines (SOLAR) package (http://www.nitrc.org/projects/se_linux). All 930 HCP subjects were included in these genetic estimations. Given the lack of detailed common environmental information for the HCP cohort, the additive genetics and unique environment (AE) model (i.e., the default model of the SOLAR) was used for both $h^{2}$ and $r_{\mathrm{g}}$ estimation. Inverse Gaussian normalization was applied to ensure the normality of WM connectivity due to the high sensitivity of SOLAR estimation to skewed distributions. To estimate the relative importance of familial and environmental effects on a phenotype, maximum likelihood variance-decomposition methods were applied, with the covariance among family members modeled as a function of genetic proximity (Almasy and Blangero 1998). Notably, the $h^{2}$ and $r_{g}$ values were re-estimated in the SOLAR without controlling for any covariates (i.e., age, gender, and hemispheric ICV), and extremely similar values were obtained (BNA: left $h^{2}, R=0.99$; right $h^{2}, R=0.99 ; r_{g}, R=0.96$; AICHA: left $h^{2}, R=0.99$; right $h^{2}, R=0.99 ; r_{g}, R=0.96$ ).

The resultant $r_{g}$ was then statistically compared with 0,1 , or -1 . Accordingly, all $r_{\mathrm{g}}$ values were stratified into 1 ) not significantly different from 0 , referred to as $r_{\mathrm{g}} \approx 0 ; 2$ ) significantly larger than 0 and significantly smaller than 1 , referred to as $0<r_{\mathrm{g}}<1$; 3) not significantly different from 1 , referred to as $r_{g} \approx 1$; 4) significantly $<0$ and significantly larger than -1 , referred to as $-1<r_{\mathrm{g}}<0$; and 5) not significantly different from -1 , referred to as $r_{\mathrm{g}} \approx-1$. The corrected $P<0.05$ (after Bonferroni correction) was set as the significance level. Given the approximate nature of the statistical results, this 5-level stratification scheme for $r_{g}$ should be considered exploratory.

To locate the distribution of the genetic influences, we projected the genetic-relevant values (i.e., $h^{2}$ value and $r_{\mathrm{g}}$ value) of WM connections to its linked regions and then averaged all projected values for each region. The resultant regional genetic measure represents the mean degree of the genetic influences of all WM connections between this region and all other connected regions. For each $r_{\mathrm{g}}$ stratification (i.e., $r_{\mathrm{g}} \approx-1,-1<r_{\mathrm{g}}<0, r_{\mathrm{g}} \approx 0$, $0<r_{\mathrm{g}}<1$, or $r_{\mathrm{g}} \approx 1$ ) based on the statistical significance, we calculated the proportion of the $r_{\mathrm{g}}$-related connections out of all WM connections for each region. To determine whether there were differences of genetic influences among functional hierarchy, we classified all BNA/AICHA regions into 5 classes according to the Mesulam's functional hierarchy scheme (Mesulam 1998), and one-way analysis of variance (ANOVA) was performed. The 5 functional hierarchies are the primary cortex, unimodal association cortex, heteromodal association cortex, paralimbic/limbic cortex, and subcortical nuclei.

\section{Results}

\section{The Heritability of WM Connectivity in the 2 Hemispheres}

As illustrated in Figure 2A, the $h^{2}$ values of WM connectivity between the hemispheres showed a great deal of variation. The spatial patterns of the 2 hemispheres appeared quite similar. Specifically, the range of $h^{2}$ values was $\sim 0-0.7$ for both hemispheres, and left and right $h^{2}$ values were strongly correlated with each other across all WM connections (BNA: $R=0.68$, $P=7.63 \times 10^{-275}$, AICHA: $R=0.72, P<1.0 \times 10^{-324}$; Fig. $\left.2 B\right)$. Furthermore, a statistical comparison showed there was no significant difference between the left and right $h^{2}$ values for any pair of homologous WM connectivities in either BNA or AICHA (corrected $P>0.05$ ).

Given the tight coupling or similarity in heritability that was observed between homologous left and right WM connectivities, we averaged the left and right $h^{2}$ values for each pair of homologous WM connections. To locate the distribution of these $h^{2}$ values, we projected the $h^{2}$ value of WM connections to its linked regions and then averaged all projected $h^{2}$ values for each region. The resultant region-specific $h^{2}$ value was taken to represent the mean heritability of WM connections between this region 
(A)

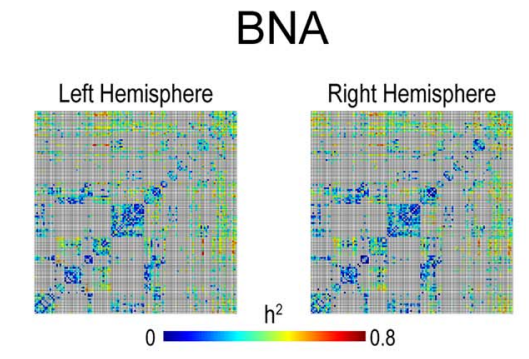

(B)

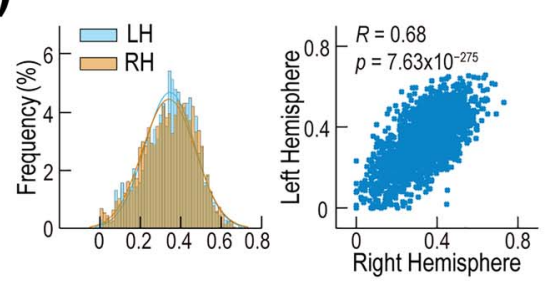

(C)

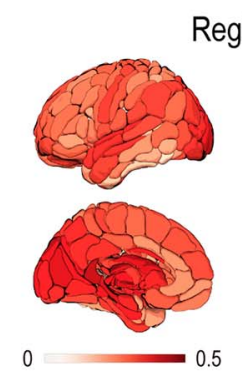

Regional $h^{2}$

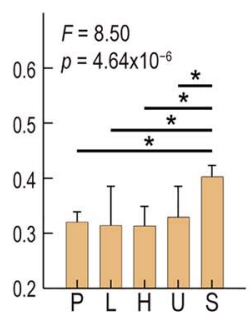

AICHA
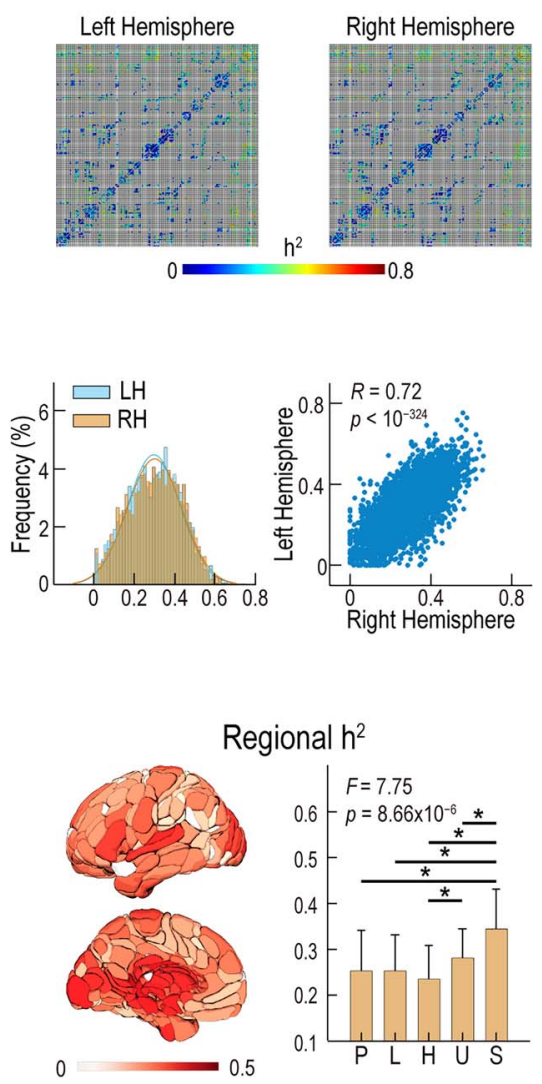

Figure 2. The heritability $\left(h^{2}\right)$ of interregional WM connectivity in the 2 hemispheres. (A) The $h^{2}$ matrices of interregional WM connectivity. Each row or column represents one BNA/AICHA region. (B) The histogram and interhemispheric scatter plot of the homologous left and right $h^{2}$. (C) The regional mean $h^{2}$ map. The 5 classes (bars), according to Mesulam's functional hierarchy scheme (Mesulam 1998). P: primary cortex; U: unimodal association cortex; H: heteromodal association cortex; L: limbic/paralimbic cortex; S: subcortical nuclei.

and all other connected regions. The whole-brain $h^{2}$ maps for both BNA and AICHA regions are illustrated in Figure $2 \mathrm{C}$, which shows that overall $h^{2}$ values were higher (i.e., darker red) for subcortical nuclei than for cortical regions. Statistical comparison (ANOVA) further revealed there was a significant difference in regional $h^{2}$ values among the 5 Mesulam's functional classes (BNA: $F_{(4,118)}=8.5, P=4.64 \times 10^{-6}$; AICHA: $F_{(4,182)}=7.75$, $P=8.66 \times 10^{-6}$; ANOVA; Fig. $2 \mathrm{C}$ ). Post hoc analysis of both the BNA and the AICHA consistently showed that the regional $h^{2}$ values were relatively higher in subcortical nuclei than in cortical classes. This indicates that WM connections originating from subcortical nuclei are more genetically controlled than the ones originating from cortical regions. For both atlases, there was no consistent difference in regional $h^{2}$ values among the 4 cortical classes (Fig. 2C).

\section{Genetic Correlations between Homologous Left and Right WM Connectivities}

Figure 3A illustrates the genetic correlations $\left(r_{\mathrm{g}}\right)$ of all homologous pairs of WM connections. The estimated $r_{\mathrm{g}}$ values ranged from -1 and 1 and showed a great deal of variation. All pairs of WM connections were then stratified into 5 levels based on statistical significance rather than raw $r_{g}$ values. Specifically, $54.9 \%$ of the pairs of BNA connections and $47.3 \%$ of the pairs of AICHA connections were statistically stratified as $r_{\mathrm{g}} \approx 1$, that is, showing a complete overlap of genetic factors underlying the homologous left and right WM connectivity. In contrast, 35.1\% of the pairs of BNA connections and $46.4 \%$ of the pairs of AICHA connections were statistically stratified as $r_{\mathrm{g}} \approx 0$, indicating a complete lack of overlap in genetic factors between the left and right WM connectivity. All remaining pairs of WM connections (10\% of paired BNA connections and $6.3 \%$ of paired AICHA connections) were statistically stratified as $0<r_{\mathrm{g}}<1$, indicating a partial overlap in genetic factors. No connection pair in either the BNA or AICHA was statistically stratified as $-1<r_{\mathrm{g}}<0$ or $r_{\mathrm{g}} \approx-1$. Clearly, both complete overlap and complete nonoverlap in the genetic factors that influence underlying homologous left and right WM connectivities are common patterns across the human brain.

To determine the $r_{\mathrm{g}}$ distribution, we also projected the $r_{\mathrm{g}}$ values of all WM connections to their connected regions to obtain a mean $r_{\mathrm{g}}$ value for each region. As shown in Figure 3B, the wholebrain $r_{\mathrm{g}}$ maps exhibited a low level of variation between regions for both the BNA and the AICHA, and there was no significant difference among the 5 classes designated according to functional hierarchy (BNA: $F_{(4,118)}=0.93 ; P=0.45 ; \operatorname{AICHA}: F_{(4,182)}=0.76$, $P=0.55$; ANOVA; Fig. $3 B$ ).

For each region, we further calculated the proportion of connections statistically stratified as $r_{\mathrm{g}} \approx 1,0<r_{\mathrm{g}}<1$, or 
(A)

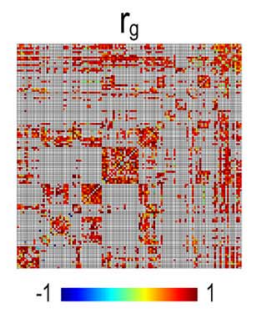

BNA

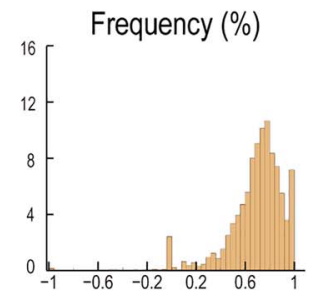

$\mathrm{AICHA}$
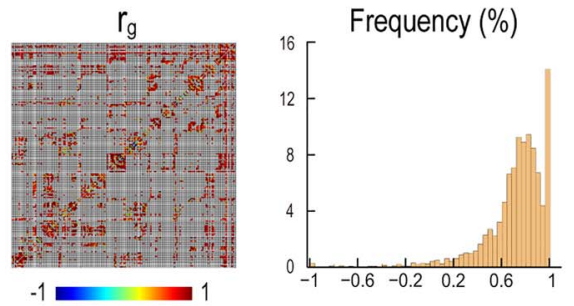

(B)

Regional $r_{g}$
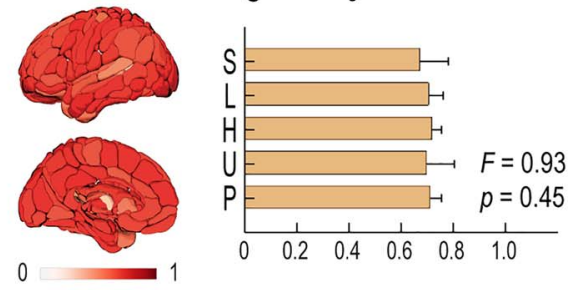

(C)

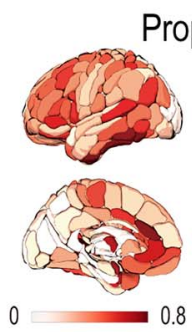

Proportion of " $\mathrm{r}_{\mathrm{g}} \approx 0$ "

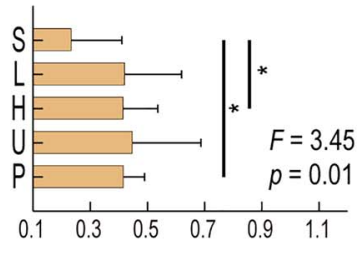

(D)

Proportion of " $0<r_{g}<1$ "
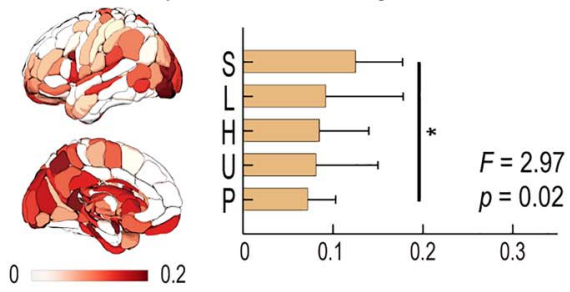

(E)

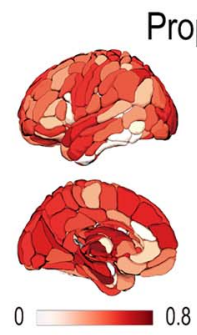

Proportion of " $\mathrm{r}_{\mathrm{g}} \approx 1$ "

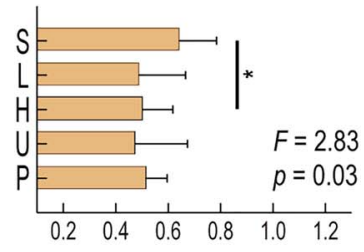

Regional $r_{g}$
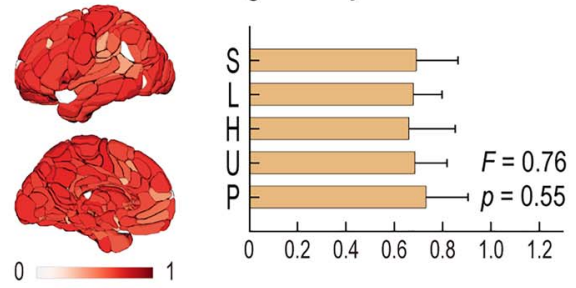

Figure 3. The genetic correlations $\left(r_{\mathrm{g}}\right)$ between homologous left and right WM connectivities of the 2 hemispheres. (A) The $r_{\mathrm{g}}$ matrices and histogram of interregional WM connectivities. Each row or column of the matrix represents one BNA/AICHI region. (B) The regional $r_{\mathrm{g}}$ map. (C) The regional map of the proportion of connections statistically stratified as $r_{\mathrm{g}} \approx 0$. (D) The regional map of the proportion of connections statistically stratified as $0<r_{\mathrm{g}}<1$. (E) The regional map of the proportion of connections statistically stratified as $r_{\mathrm{g}} \approx 1$. The 5 classes (bars) were defined according to Mesulam's functional hierarchy scheme (Mesulam 1998). 
$r_{\mathrm{g}} \approx 0$ out of all WM connections of the region (Fig. 3CE). Interestingly, there was a significant difference in the proportions of connections statistically stratified as $r_{\mathrm{g}} \approx 1$ and $r_{\mathrm{g}} \approx 0$ among the 5 classes separated according to functional hierarchy (as shown in Fig. $3 C, r_{\mathrm{g}} \approx 0$ : BNA, $F_{(4,118)}=3.45, P=0.01$; AICHA, $F_{(4,182)}=8.12, P=4.79 \times 10^{-6}$; as shown in Fig. $3 E, r_{\mathrm{g}} \approx 1$ : $\mathrm{BNA}, F_{(4,118)}=2.83, \mathrm{P}=0.03$; AICHA, $F_{(4,182)}=8.51, P=2.57 \times 10^{-6}$; ANOVA). Specifically, compared with cortical regions, subcortical nuclei had a higher proportion of connections stratified as $r_{\mathrm{g}} \approx 1$ but a lower proportion of connections stratified as $r_{\mathrm{g}} \approx 0$. Regarding the proportion of connections stratified as $0<r_{\mathrm{g}}<1$, they were significantly different only for the BNA but not for the AICHA (as shown in Fig. 3D, BNA, $F_{(4,118)}=2.97, P=0.02$; AICHA, $F_{(4,182)}=0.07, P=0.99 ;$ ANOVA). These results collectively indicate that homologous left and right WM connections from subcortical nuclei were more likely to have completely overlapping patterns of genetic factors and less likely to have a nonoverlapping pattern of genetic factors. Notably, the observed significant results for the proportion of stratified connections contrasted with the negative results for the raw $r_{g}$ values above, suggesting that the 2 measures capture related but distinct aspects of genetic correlation between homologous WM connectivity.

\section{Effect of Connectional Length on Heritability and Interhemispheric Genetic Correlations}

All pairs of homologous left and right WM connections were categorized as short- or long-range based on the streamline length (BNA, 947/1088 short-/long-range connections; AICHA, 1430/1410 short-/long-range connections) and Euclidean distance (BNA, 1067/968 short-/long-range connections; AICHA, 1598/1242 short-/long-range connections). In addition, anatomically within-system (i.e., short-range) and between-system (i.e., long-range) connections were adopted as additional criteria for categorizing connections (BNA, 704/1331 within-/betweensystem connections; AICHA, 1112/1728 within-/between-system connections). Across the 3 short-/long-range criteria, longrange connections consistently had significantly higher $h^{2}$ and $r_{g}$ values than were found for short-range connections (Fig. $4 \mathrm{~A}$ and $\mathrm{B}, \mathrm{h}^{2}$ : all $\mathrm{P}<=1.61 \times 10^{-79}$; Fig. $4 \mathrm{C}$ and $\mathrm{D}, \mathrm{r}_{\mathrm{g}}$ : all $P<=0.005$, degree of freedom: 2033 for BNA, 2838 for AICHA). In line with these $r_{\mathrm{g}}$ value-related results, the proportions of connections statistically stratified as $r_{\mathrm{g}} \approx 1,0<r_{\mathrm{g}}<1$, and $r_{\mathrm{g}} \approx 0$ were significantly different between long- and shortrange connections ( $\chi^{2}$ test: all $P \leq 3.41 \times 10^{-60}$ ), with longrange connections having a higher proportion of connections statistically stratified as $r_{\mathrm{g}} \approx 1$ and a lower proportion of connections statistically stratified as $r_{\mathrm{g}} \approx 0$. Moreover, we compared the longest 500 connections with the shortest 500 connections and found the same significant results (data not shown). Notably, the effect size of the observed length effect was remarkably large for heritability but relatively small for genetic correlations (Fig. 4).

It is possible that these length effects are relevant to or simply responsible for the above-observed higher $h^{2}$ values and the proportion of connections statistically stratified as $r_{\mathrm{g}} \approx 1$ of subcortical nuclei. To evaluate this possibility, we compared these measures between connections originating from subcortical nuclei (referred to as subcortical connections) and connections between cortical regions (referred to as within-cortical connections) after controlling for connectional length. Higher $h^{2}$ remained significantly higher for subcortical connections (for both atlases and both length definitions, all $\mathrm{T} \leq-15.17$, all $P \leq 4.58 \times 10^{-50}$; degree of freedom: 2032 for BNA, 2837 for AICHA). For both short- and long-range connections, we also found that the proportion of connections statistically stratified as $r_{\mathrm{g}} \approx 1$ was significantly higher and the proportion of connections statistically stratified as $r_{\mathrm{g}} \approx 0$ lower for subcortical nuclei (all $\chi^{2} \geq 11.71$, all $P \leq 0.0029$ ). Thus, the $h^{2}$ - and $r_{\mathrm{g}}$-related differences between subcortical and within-cortical connections cannot simply be attributed to the length effect on these measures.

We next assessed whether there are also significant length effects on $h^{2}$ and $r_{\mathrm{g}}$ when considering subcortical connections alone or within-cortical connections alone. The relevant results are summarized in Supplementary Figures S1 and S2, and the vast majority of the length effects (i.e., higher $h^{2}$ and $r_{g}$ values and the proportion of connections statistically stratified as $r_{\mathrm{g}} \approx 1$ for long-range connections) remained in either subcortical connections only or within-cortical connections only. This result implies that there is a general length effect on the genetics of WM connectivity across the connection sets of the entire hemisphere, the subcortical system, and the cortical system.

Finally, to evaluate the reproducibility of our results, we reran the main analyses using split-half samples. Specifically, all 930 HCP subjects were randomly divided into 2 subsamples that were matched in the number of monozygotic twins. The 2 subsamples (each subsample: 465 subjects) yielded highly reproducible results that were similar to our key findings achieved when using the full HCP samples, as described above. All splithalf results are included in the Supplementary Materials.

\section{Discussion}

In the present study, we use human twin and pedigree models to reveal, for the first time, the patterns of heritability and genetic correlations of the homologous interregional WM connectivities of the 2 hemispheres. Our results demonstrate that the heritability of WM connectivities was quite similar and coupled between the 2 hemispheres and that both complete overlap and complete nonoverlap in the genetic factors underlying homologous left and right WM connectivities are common across the human brain. The degree of heritability was significantly higher and the chance of interhemispheric complete overlap in genetic factors was higher in subcortical WM connections than in cortical WM connections. In particular, there were length effects on the genetics of homologous left and right WM connectivity, with heritability higher and interhemispheric genetic correlations stronger for long-range connections than for short-range connections.

Importantly, the present study used 2 distinct atlases that were derived using WM connectivity (BNA) and resting-state functional connectivity profiles (AICHA), respectively. While the BNA is theoretically more suitable than AICHA for our study, the results from both atlases are highly reproducible, indicating the robustness and generalizability of our findings.

\section{Genetic Relationships between Homologous Left and Right WM Connectivities}

Many previous studies have shown that there is no difference in heritability between the 2 hemispheres (Wright et al. 2002; Eyler et al. 2011a; Eyler et al. 2014; Wen et al. 2016; Liu et al. 2019; Zhao et al. 2019a), whereas a few others have contrastingly reported finding hemispheric differences in heritability 
(A)

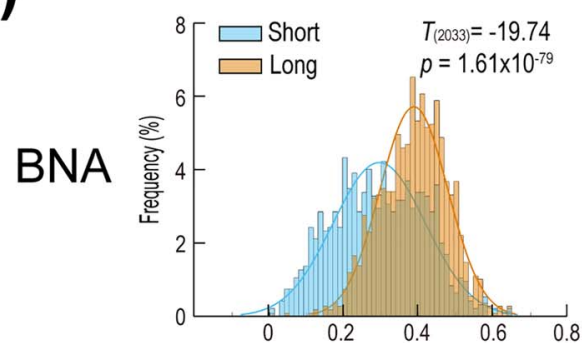

Euclidean

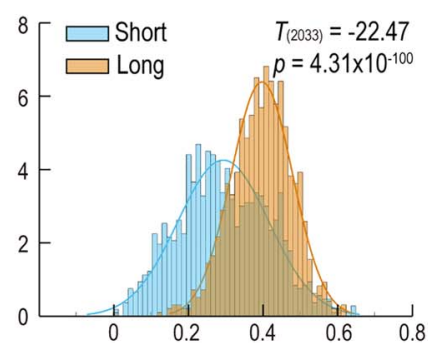

System

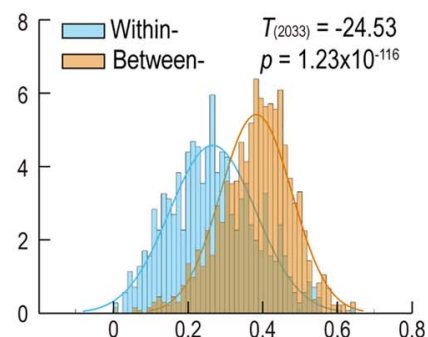

(B)

$\mathrm{AICHA}$
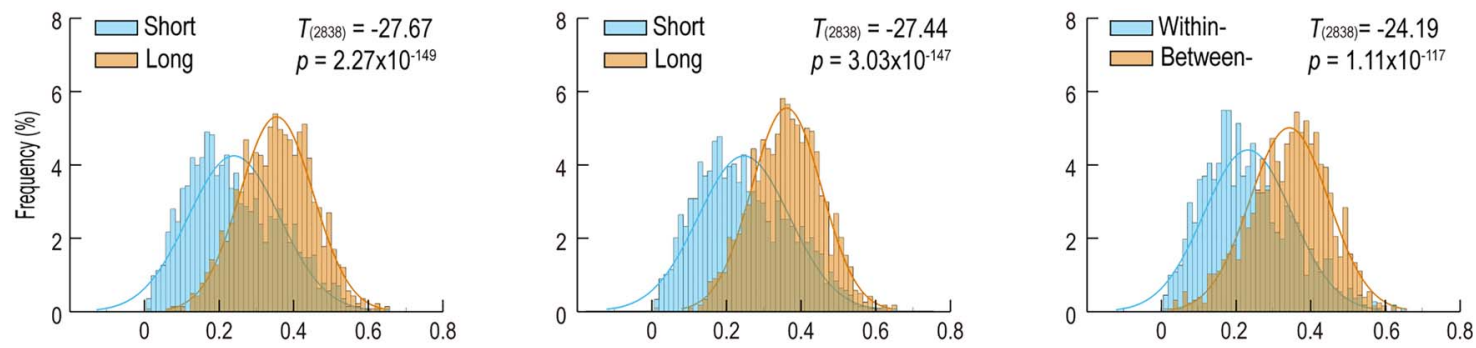

(C)
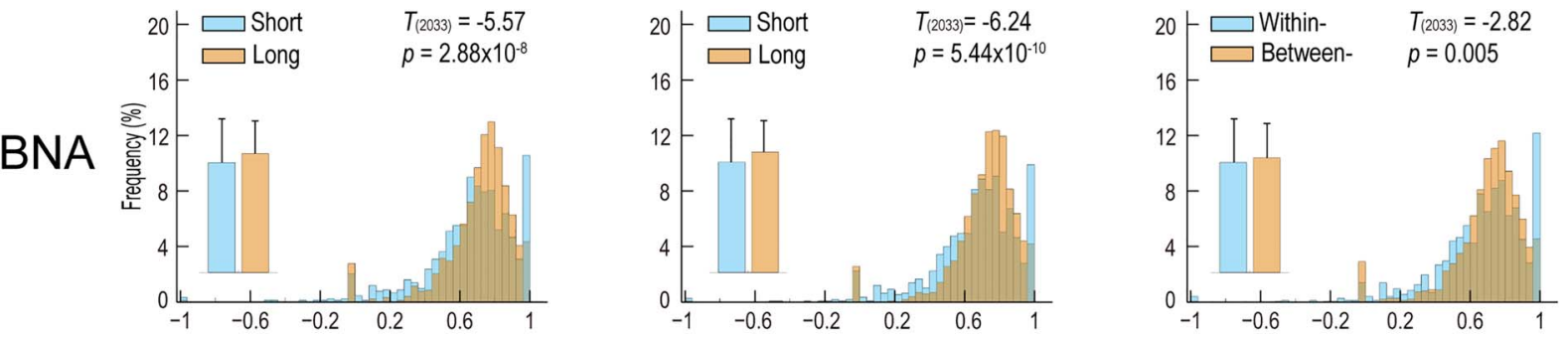

(D)
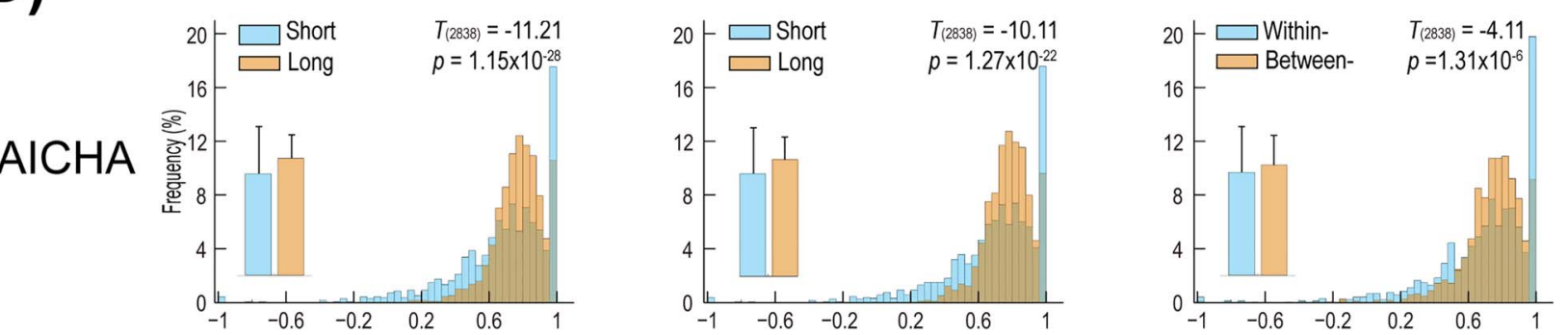

Figure 4. The difference in heritability $\left(h^{2}\right)$ and interhemispheric genetic correlations $\left(r_{\mathrm{g}}\right)$ between short- and long-range connections. (A) The mean $h^{2}$ of homologous left and right WM connectivities for short- and long-range connections of the BNA. (B) The mean $h^{2}$ of homologous left and right WM connectivities for short- and long-range connections of the AICHA. (C) The $r_{\mathrm{g}}$ for short- and long-range connections of the BNA. (D) The $r_{\mathrm{g}}$ for short- and long-range connections of the AICHA. The 3 columns represent the categorization of short- and long-range connections based on streamline length, Euclidean distance, and anatomical system, respectively.

(Thompson et al. 2001; Geschwind et al. 2002; Yoon et al. 2010). Notably, the reported hemispheric differences in heritability were found in studies with very small sample sizes or a lack of statistical tests to identify such a difference, as summarized by Eyler and colleagues (Eyler et al. 2014). Our observed result of indistinguishable heritability for any pair of homologous left and right WM connections is complementary to those presented in previous studies, which have mostly explored GM phenotypes, thus providing joint support for general bilateral similarities in heritability for human brain structural phenotypes.

In addition to the paired homologous WM connections of the 2 hemispheres, we estimated the heritability of connections that exist only in one hemisphere. These connections manifest comparable heritability with the homologous WM connection 
pairs, and reflect certain degree phenotypic laterality of WM connectivity. Intriguingly, we observed higher heritability of cross-hemispheric connections, relative to within-hemispheric connections (see Supplementary Materials). The genetics underlying the nonpaired WM connections warrants further investigation.

Notably, similar bilateral heritability does not necessarily mean that similar genetic factors underlie homologous left and right WM connectivities. To quantify the degree to which genetic factors overlap between 2 phenotypes, one could estimate the genetic correlation (Carey 1988). In previous studies that measured the genetic correlations in various GM phenotypes between regions, the homologous regions in the 2 hemispheres have always shown very strong genetic correlations (Eyler et al. 2011b; Chen et al. 2012; Chen et al. 2013; Eyler et al. 2014; Wen et al. 2016; Liu et al. 2019), suggesting that the genetic factors underlying individual differences in one hemisphere overlap with those that influence the other hemisphere. In line with these findings related to GM phenotypes, we observed that $>50 \%$ of pairs of left and right WM connections were statistically indistinguishable from a genetic correlation $=1$, which indicates that identical genetic factors underlie the development of the homologous left and right connections. This overlap in genetic factors underlying homologous left and right WM connections likely contributes to the bilateral abnormalities in hemispheric WM connections observed in patients with congenital genetic deficits (Ganou and Grouios 2008; Zhao et al. 2019b). In addition, the synchronized growth or degeneration of WM in the 2 hemispheres might partially be attributed to this genetic overlap.

However, a substantial proportion of connection pairs were significantly different from a genetic correlation $=1$, suggesting that substantially unique or different genetic factors influence the development of homologous left and right WM connectivities. In fact, previous GM-related studies have also shown that the left-right genetic correlations of particular regions are significantly different from 1 (Guadalupe et al. 2016; Kong et al. 2018). Unexpectedly, the majority of these pairs of WM connections between the 2 hemispheres are statistically indistinguishable from a genetic correlation $=0$, indicating a complete lack of overlap in underlying genetic factors and a strong genetic asymmetry. For trait pairs, there is no predictable relation between genetic correlation and phenotypic correlation, for example, a stronger genetic correlation does not necessarily indicate a stronger phenotypic correlation, and vice versa. Empirically, however, our data revealed a significantly positive correlation between genetic correlation and phenotypic correlation across homologous connectivity pairs $(r=0.53)$. This observation deserves exclusive investigation in the future.

\section{Genetic Differences between Subcortical and Cortical WM Connectivity}

In terms of heritability and interhemispheric genetic correlations, we show that WM connections belonging to the subcortical and cortical systems are genetically different, a finding that cannot simply be attributed to the observed length effect. The higher heritability of subcortical WM connectivity is consistent with the previously reported higher heritability of subcortical GM volume relative to cortical GM volume (Blokland et al. 2012; van der Lee et al. 2017), and these findings jointly imply that there is stronger genetic control of subcortical system. Notably, this observation is compatible with classical notions of the subcortical system: 1) Subcortical nuclei are evolutionarily more ancient and conserved (Satizabal et al. 2019) and 2) Neurogenesis and development occur earlier in subcortical nuclei than in the cerebral neocortex (Van Essen et al. 2018). Regarding genetic correlation, Wen and colleagues recently found that GM structures within the cortical or subcortical systems are genetically clustered together, supporting that distinct genetic factors underlie the development of these 2 cerebral systems (Wen et al. 2016). As a further step, we observed that the chance of interhemispheric complete overlap in genetic factors (i.e., $r_{g}=1$ ) was higher for subcortical WM connections, indicating that genetic coupling is stronger for the bilateral subcortical systems of the 2 hemispheres than for bilateral cortical systems. Notably, this observation is based on the proportion of connections that is stratified using the statistical significance of genetic correlation, and direct comparison on genetic correlation did not show a significant difference.

Given the extensively described evolutionary and functional differences between the primary/limbic/paralimbic and association cortices (Sousa et al. 2017; Van Essen et al. 2018), it is plausible to expect that there would be genetic differences between these cortical systems. Our results, however, do not show such a difference in either heritability or interhemispheric genetic correlations. This might be related to the small effect size of these genetic measures between these cortical subsystems. Alternatively, it is also possible that WM connectivity is simply unaffected by genetic differences between these cortical subsystems.

\section{Length Effects on the Genetics of Homologous Left and Right WM Connectivity}

Length is an important characteristic of WM connections (Kaiser 2017). WM connections with different lengths are nonrandomly distributed across the entire brain, and connectivity strength has an exponentially negative relationship with length (Ercsey-Ravasz et al. 2013). In contrast to short-range connections, long-range connections are costly in terms of the consumption of physical space and energy (Bullmore and Sporns 2012). However, it has been proposed that long-range connections are necessary within brain networks to increase communicating efficiency because they reduce the number of processing paths between areas (Sporns and Zwi 2004; Kaiser and Hilgetag 2006) or enhance the diversity of inputs and outputs of brain areas, thereby supporting complex brain dynamics (Betzel and Bassett 2018). The differences observed in this study in the heritability and interhemispheric genetic correlations between short- and long-range WM connections provide strong genetic support for the proposed system-level functional distinction between them.

Specifically, long-range WM connections showed a stronger degree of genetic control and more overlap in genetic factors between the 2 hemispheres. This pattern is compatible with the putative role of long-range connections as the predetermined backbones of brain networks (Kaiser 2017). Accordingly, environmental and experience-related factors can be inferred to have stronger impacts on short-range connections, implying that these connections have more developmental plasticity. Compatibly with this, development of fiber myelination was reported later for short-range tracts (Giedd et al., 1999; Ouyang et al., 2017), and later-developing brain structures are putatively more environmentally influenced (Lohmann et al., 1999; Brun et al., 2009). This heritability difference may also relate to distinct abnormalities of long and short connections in brain diseases 
(e.g., autism and schizophrenia) (Nazeri et al. 2013; Ameis and Catani 2015).

Finally, the findings presented in our present study provide general insight into the origin of interhemispheric differences and coupling between left and right WM connectivities. For instance, if there is a strong interhemispheric genetic correlation for a particular phenotype, it suggests low heritability for asymmetry (Eyler et al. 2014). Accordingly, those WM connections with strong interhemispheric genetic correlations should have a low degree of heritability for asymmetries, in agreement with repeated reports showing zero-to-modest heritability values for various brain asymmetries (Francks 2015). Specifically, given the higher genetic correlations of subcortical WM connections or long-range WM connections, asymmetries in these connections should therefore be statistically less heritable, implying environmental factors and postnatal experiences play a dominant role in the asymmetries of these connections. In addition, the genetic contribution to observed phenotypic correlations for any 2 phenotypes is the product of their genetic correlation and both of their degrees of heritability; hence, phenotypic interhemispheric correlations/couplings in subcortical WM connections or long-range WM connections that are observed across individuals should be largely attributed to underlying genetic factors, given that these connections have both higher heritability and strong genetic correlations. These implications are informative for interpreting results regarding the asymmetries or covariation of WM connectivities in both healthy and disease states, and could inspire new investigations along this line.

\section{Limitations}

There are several caveats to the present study. First, our current analyses are confined to WM connectivity, and the analysis framework can be directly applied to reveal the interhemispheric genetic relation of functional connectivity in the future. Particularly, our observed interhemispheric genetic relations represent system-level patterns across the entire hemisphere, and they should not be simply generalized to individual WM connections (e.g., one cannot conclude that all long/subcortical connections have strong heritability and genetic correlations). Next, our findings are based on data of the young adult HCP cohort, in which the actual sample size of twins is moderate. Our results therefore need to be further verified using a completely independent data set with a larger number of twins. Given the previously reported age dependence of the heritability of various brain phenotypes (Blokland et al. 2012; Schmitt et al. 2014; Jansen et al. 2015), caution should also be exercised when extrapolating our findings across the lifespan. Finally, considering the inability of our diffusion tractography to accommodate for strong geometric complexity within voxels, the tractographybased connectivity metrics decline especially within the voxels near the cortex. Thus, these connectivity measures, especially near the cortex, should be viewed with degree of uncertainty.

\section{Supplementary Material}

Supplementary material is available at Cerebral Cortex online.

\section{Funding}

National Science Foundation of China (81671772, 91732101 to G.G., 81701783 to S.Z.), the Research Fund of PLA of China (AWS17J011, G.G.), and the Fundamental Research Funds for the
Central Universities. C.F. was funded by the Max Planck Society (Germany).

\section{Notes}

Data were provided by the Human Connectome Project, WUMinn Consortium (Principal Investigators: David Van Essen and Kamil Ugurbil; 1U54MH091657) funded by the 16 National Institutes of Health and Centers that support the National Institutes of Health Blueprint for Neuroscience Research and by the McDonnell Center for Systems Neuroscience at Washington University. Conflict of Interest: None declared.

\section{References}

Almasy L, Dyer TD, Blangero J. 1997. Bivariate quantitative trait linkage analysis: pleiotropy versus co-incident linkages. Genet Epidemiol. 14:953-958.

Almasy L, Blangero J. 1998. Multipoint quantitative-trait linkage analysis in general pedigrees. Am J Hum Genet. 62:1198-1211.

Ameis SH, Catani M. 2015. Altered white matter connectivity as a neural substrate for social impairment in autism Spectrum disorder. Cortex. 62:158-181.

Andescavage NN, du Plessis A, McCarter R, Serag A, Evangelou I, Vezina G, Robertson R, Limperopoulos C. 2016. Complex trajectories of brain development in the healthy human fetus. Cereb Cortex. 27:5274-5283.

Betzel RF, Bassett DS. 2018. Specificity and robustness of longdistance connections in weighted, interareal connectomes. Proc Natl Acad Sci U S A. 115:E4880-E4889.

Bishop DV. 2013. Cerebral asymmetry and language development: cause, correlate, or consequence? Science. 340:1230531.

Blokland GA, de Zubicaray GI, McMahon KL, Wright MJ. 2012. Genetic and environmental influences on neuroimaging phenotypes: a meta-analytical perspective on twin imaging studies. Twin Res Hum Genet. 15:351-371.

Brun CC, Leporé N, Pennec X, Lee AD, Barysheva M, Madsen SK, Avedissian C, Chou YY, de Zubicaray GI, McMahon $\mathrm{KL}$, et al. 2009. Mapping the regional influence of genetics on brain structure variability-a tensor-based morphometry study. Neuroimage. 48:37-49.

Bullmore E, Sporns O. 2012. The economy of brain network organization. Nat Rev Neurosci. 13:336-349.

Carey G. 1988. Inference about genetic correlations. Behav Genet. 18:329-338.

Chen C-H, Gutierrez ED, Thompson W, Panizzon MS, Jernigan TL, Eyler LT, Fennema-Notestine C, Jak AJ, Neale MC, Franz $\mathrm{CE}$, et al. 2012. Hierarchical genetic organization of human cortical surface area. Science. 335:1634-1636.

Chen C-H, Fiecas M, Gutiérrez ED, Panizzon MS, Eyler LT, Vuoksimaa E, Thompson WK, Fennema-Notestine C, Hagler DJ Jr, Jernigan TL, et al. 2013. Genetic topography of brain morphology. Proc Natl Acad Sci U S A. 110:17089-17094.

Chiang M-C, McMahon KL, de Zubicaray GI, Martin NG, Hickie I, Toga AW, Wright MJ, Thompson PM. 2011. Genetics of white matter development: a DTI study of 705 twins and their siblings aged 12 to 29. Neuroimage. 54:2308-2317.

Dhollander T, Raffelt D, Connelly A. 2016. Unsupervised 3-tissue response function estimation from single-shell or multi-shell diffusion MR data without a co-registered T1 image. In: Proceedings of ISMRM Workshop on Breaking the Barriers of Diffusion MRI. Lisbon, Portugal, p. 5. 
Donahue CJ, Sotiropoulos SN, Jbabdi S, Hernandez-Fernandez M, Behrens TE, Dyrby TB, Coalson T, Kennedy H, Knoblauch K, Van Essen DC, et al. 2016. Using diffusion tractography to predict cortical connection strength and distance: a quantitative comparison with tracers in the monkey. J Neurosci. 36:6758-6770.

Ercsey-Ravasz M, Markov Nikola T, Lamy C, Van Essen David C, Knoblauch K, Toroczkai Z, Kennedy H. 2013. A predictive network model of cerebral cortical connectivity based on a distance rule. Neuron. 80:184-197.

Eyler LT, Prom-Wormley E, Panizzon MS, Kaup AR, FennemaNotestine C, Neale MC, Jernigan TL, Fischl B, Franz CE, Lyons MJ. 2011a. Genetic and environmental contributions to regional cortical surface area in humans: a magnetic resonance imaging twin study. Cereb Cortex. 21: 2313-2321.

Eyler LT, Prom-Wormley E, Fennema-Notestine C, Panizzon MS, Neale MC, Jernigan TL, Fischl B, Franz CE, Lyons MJ, Stevens A. 2011b. Genetic patterns of correlation among subcortical volumes in humans: results from a magnetic resonance imaging twin study. Hum Brain Mapp. 32:641-653.

Eyler LT, Vuoksimaa E, Panizzon MS, Fennema-Notestine C, Neale MC, Chen C-H, Jak A, Franz CE, Lyons MJ, Thompson WK. 2014. Conceptual and data-based investigation of genetic influences and brain asymmetry: a twin study of multiple structural phenotypes. J Cogn Neurosci. 26: 1100-1117.

Fan L, Li H, Zhuo J, Zhang Y, Wang J, Chen L, Yang Z, Chu C, Xie S, Laird AR, et al. 2016. The human Brainnetome atlas: a new brain atlas based on connectional architecture. Cereb Cortex. 26:3508-3526.

Francks C. 2015. Exploring human brain lateralization with molecular genetics and genomics. Ann N Y Acad Sci. 1359:1-13.

Ganou M, Grouios G. 2008. Cerebral laterality in turner syndrome: a critical review of the literature. Child Neuropsychol. 14:135-147.

Geschwind DH, Miller BL, DeCarli C, Carmelli D. 2002. Heritability of lobar brain volumes in twins supports genetic models of cerebral laterality and handedness. Proc Natl Acad Sci U S A. 99:3176-3181.

Giedd JN, Blumenthal J, Jeffries NO, Castellanos FX, Liu H, Zijdenbos A, Paus T, Evans AC, Rapoport JL. 1999. Brain development during childhood and adolescence: a longitudinal MRI study. Nat Neurosci. 2:861-863.

Glasser MF, Sotiropoulos SN, Wilson JA, Coalson TS, Fischl B, Andersson JL, Xu J, Jbabdi S, Webster M, Polimeni JR, et al. 2013. The minimal preprocessing pipelines for the Human Connectome Project. Neuroimage. 80:105-124.

Gong G, He Y, Concha L, Lebel C, Gross DW, Evans AC, Beaulieu C. 2009. Mapping anatomical connectivity patterns of human cerebral cortex using in vivo diffusion tensor imaging tractography. Cereb Cortex. 19:524-536.

Guadalupe T, Mathias SR, vanErp TG, Whelan CD, Zwiers MP, Abe Y, Abramovic L, Agartz I, Andreassen OA, Arias-Vasquez A, et al. 2016. Human subcortical brain asymmetries in 15,847 people worldwide reveal effects of age and sex. Brain Imaging Behav. 11:1497-1514.

Hänggi J, Fövenyi L, Liem F, Meyer M, Jäncke L. 2014. The hypothesis of neuronal interconnectivity as a function of brain sizea general organization principle of the human connectome. Front Hum Neurosci. 8:915.
Herve PY, Zago L, Petit L, Mazoyer B, Tzourio-Mazoyer N. 2013. Revisiting human hemispheric specialization with neuroimaging. Trends Cogn Sci. 17:69-80.

Jansen AG, Mous SE, White T, Posthuma D, Polderman TJ. 2015. What twin studies tell us about the heritability of brain development, morphology, and function: a review. Neuropsychol Rev. 25:27-46.

Jeurissen B, Tournier J-D, Dhollander T, Connelly A, Sijbers J. 2014. Multi-tissue constrained spherical deconvolution for improved analysis of multi-shell diffusion MRI data. Neuroimage. 103:411-426.

Joliot M, Jobard G, Naveau M, Delcroix N, Petit L, Zago L, Crivello F, Mellet E, Mazoyer B, Tzourio-Mazoyer N. 2015. AICHA: an atlas of intrinsic connectivity of homotopic areas. J Neurosci Methods. 254:46-59.

Kaiser M, Hilgetag CC. 2006. Non-optimal component placement, but short processing paths, due to long-distance projections in neural systems. PLoS Comput Biol. 12:e95.

Kaiser M. 2017. Mechanisms of connectome development. Trends Cogn Sci. 21:703-717.

Kochunov P, Jahanshad N, Sprooten E, Nichols TE, Mandl RC, Almasy L, Booth T, Brouwer RM, Curran JE, de Zubicaray GI, et al. 2014. Multi-site study of additive genetic effects on fractional anisotropy of cerebral white matter: comparing meta and megaanalytical approaches for data pooling. Neuroimage. 95:136-150.

Kochunov P, Jahanshad N, Marcus D, Winkler A, Sprooten E, Nichols TE, Wright SN, Hong LE, Patel B, Behrens T, et al. 2015. Heritability of fractional anisotropy in human white matter: a comparison of Human Connectome Project and ENIGMA-DTI data. Neuroimage. 111:300-311.

Kong XZ, Mathias SR, Guadalupe T, ELW G, Glahn DC, Franke B, Crivello F, Tzourio-Mazoyer N, Fisher SE, Thompson PM, et al. 2018. Mapping cortical brain asymmetry in 17,141 healthy individuals worldwide via the ENIGMA consortium. Proc Natl Acad Sci U S A. 115:E5154-E5163.

Kremen WS, Prom-Wormley E, Panizzon MS, Eyler LT, Fischl B, Neale MC, Franz CE, Lyons MJ, Pacheco J, Perry ME. 2010. Genetic and environmental influences on the size of specific brain regions in midlife: the VETSA MRI study. Neuroimage. 49:1213-1223.

Le Guen Y, Auzias G, Leroy F, Noulhiane M, Dehaene-Lambertz G, Duchesnay E, Mangin J, Coulon O, Frouin V. 2018. Genetic influence on the sulcal pits: on the origin of the first cortical folds. Cereb Cortex. 28:1922-1933.

Lee SJ, Steiner RJ, Luo S, Neale MC, Styner M, Zhu H, Gilmore JH. 2015. Quantitative tract-based white matter heritability in twin neonates. Neuroimage. 111:123-135.

Liu S, Li A, Zhu M, Li J, Liu B. 2019. Genetic influences on cortical myelination in the human brain. Genes Brain Behav. 18:e12537.

Lohmann G, von Cramon DY, Steinmetz H. 1999. Sulcal variability of twins. Cereb Cortex. 9:754-763.

Mesulam MM. 1998. From sensation to cognition. Brain. 121:1013-1052.

Nazeri A, Chakravarty MM, Felsky D, Lobaugh NJ, Rajji TK, Mulsant BH, Voineskos AN. 2013. Alterations of superficial white matter in schizophrenia and relationship to cognitive performance. Neuropsychopharmacology. 38:1954-1962.

Neale MC, Cardon LR. 1992. Methodology for genetic studies of twins and families. Dordrecht (Netherlands): Kluwer Academic.

Neale MC, Miller MB. 1997. The use of likelihood-based confidence intervals in genetic models. Behav Genet. 27:113-120. 
Ouyang M, Kang H, Detre JA, Roberts TPL, Huang H. 2017. Shortrange connections in the developmental connectome during typical and atypical brain maturation. Neurosci Biobehav Rev. 83:109-122.

Satizabal CA-O, Adams HA-O, Hibar DP, White CC, Knol MAO, Stein JL, Scholz MA-O, Sargurupremraj M, Jahanshad N, Roshchupkin GA-O, et al. 2019. Genetic architecture of subcortical brain structures in 38,851 individuals. Nat Genet. 51:1624-1636.

Schmitt JE, Neale MC, Fassassi B, Perez J, Lenroot RK, Wells EM, Giedd JN. 2014. The dynamic role of genetics on cortical patterning during childhood and adolescence. Proc Natl Acad Sci U S A. 111:6774-6779.

Scott JA, Hamzelou KS, Rajagopalan V, Habas PA, Kim K, Barkovich AJ, Glenn OA, Studholme C. 2012. 3D morphometric analysis of human fetal cerebellar development. Cerebellum. 11:761-770.

Smith RE, Tournier JD, Calamante F, Connelly A. 2012. Anatomically-constrained tractography: improved diffusion MRI streamlines tractography through effective use of anatomical information. Neuroimage. 62:1924-1938.

Smith RE, Tournier JD, Calamante F, Connelly A. 2013. SIFT: spherical-deconvolution informed filtering of tractograms. Neuroimage. 67:298-312.

Smith SM, Jenkinson M, Woolrich MW, Beckmann CF, Behrens TE, Johansen-Berg H, Bannister PR, De Luca M, Drobnjak I, Flitney DE, et al. 2004. Advances in functional and structural MR image analysis and implementation as FSL. Neuroimage. 23(Suppl 1):S208-S219.

Sotiropoulos SN, Zalesky A. 2019. Building connectomes using diffusion MRI: why, how and but. NMR Biomed. 32: e3752.

Sousa AMM, Meyer KA, Santpere G, Gulden FO, Sestan N. 2017. Evolution of the human nervous system function, structure, and development. Cell. 170:226-247.

Sporns O, Zwi JD. 2004. The small world of the cerebral cortex. Neuroinformatics. 2:145-162.

Sporns O. 2011. The human connectome: a complex network. Ann N Y Acad Sci. 1224:109-125.

Thompson PM, Cannon TD, Narr KL, Van Erp T, Poutanen V-P, Huttunen M, Lönnqvist J, Standertskjöld-Nordenstam C-G, Kaprio J, Khaledy M. 2001. Genetic influences on brain structure. Nat Neurosci. 4:1253-1258.

Thompson PM, Ge T, Glahn DC, Jahanshad N, Nichols TE. 2013. Genetics of the connectome. Neuroimage. 80:475-488.

Toga AW, Thompson PM. 2003. Mapping brain asymmetry. Nat Rev Neurosci. 4:37-48.
Tournier JD, Calamante F, Connelly A. 2007. Robust determination of the fibre orientation distribution in diffusion MRI: non-negativity constrained super-resolved spherical deconvolution. Neuroimage. 35:1459-1472.

Tournier JD, Calamante F, Connelly A. 2012. MRtrix: diffusion tractography in crossing fiber regions. Int J Imag Syst Tech. 22:53-66.

Tournier JD, Smith R, Raffelt D, Tabbara R, Dhollander T, Pietsch M, Christiaens D, Jeurissen B, Yeh CH, Connelly A. 2019. MRtrix3: a fast, flexible and open software framework for medical image processing and visualisation. Neuroimage. 202:116137.

van der Lee SJ, Roshchupkin GV, Adams HH, Schmidt H, Hofer E, Saba Y, Schmidt R, Hofman A, Amin N, van Duijn CM, et al. 2017. Gray matter heritability in family-based and population-based studies using voxel-based morphometry. Hum Brain Mapp. 38:2408-2423.

Van Essen DC, Ugurbil K, Auerbach E, Barch D, Behrens TE, Bucholz R, Chang A, Chen L, Corbetta M, Curtiss SW, et al. 2012. The Human Connectome Project: a data acquisition perspective. Neuroimage. 62:2222-2231.

Van Essen DC, Donahue CJ, Glasser MF. 2018. Development and evolution of cerebral and cerebellar cortex. Brain Behav Evol. 91:158-169.

Wen W, Thalamuthu A, Mather KA, Zhu W, Jiang J, de Micheaux PL, Wright MJ, Ames D, Sachdev PS. 2016. Distinct genetic influences on cortical and subcortical brain structures. Sci Rep. 6:32760.

Wright IC, Sham P, Murray RM, Weinberger DR, Bullmore ET. 2002. Genetic contributions to regional variability in human brain structure: methods and preliminary results. Neuroimage. 17:256-271.

Yoon U, Fahim C, Perusse D, Evans AC. 2010. Lateralized genetic and environmental influences on human brain morphology of 8-year-old twins. Neuroimage. 53:1117-1125.

Zhao B, Ibrahim JG, Li Y, Li T, Wang Y, Shan Y, Zhu Z, Zhou F, Zhang J, Huang C, et al. 2019a. Heritability of regional brain volumes in large-scale neuroimaging and genetic studies. Cereb Cortex. 29:2904-2914.

Zhao C, Yang L, Xie S, Zhang Z, Pan H, Gong G. 2019b. Hemispheric module-specific influence of the $\mathrm{X}$ chromosome on White matter connectivity: evidence from girls with turner syndrome. Cereb Cortex. 29:4580-4594.

Zhong S, He Y, Shu H, Gong G. 2017. Developmental changes in topological asymmetry between hemispheric brain White matter networks from adolescence to young adulthood. Cereb Cortex. 27:2560-2570. 